\title{
Correlated Response to Phototactic Selection
}

\author{
Therese Ann Markow ${ }^{1}$ and Andrew G. Clark ${ }^{2}$
}

Received 10 Aug. 1983-Final 8 Mar. 1984

\begin{abstract}
Artificial selection for positive and negative phototaxis was conducted in populations of Drosophila melanogaster that were polymorphic at the sepia locus. Photoselection response was accompanied by a correlated response in the frequency of the sepia allele. Changes in sepia frequency were shown to be significantly different from those predicted by several neutral models. Implications of this correlated response are briefly discussed in terms of the neurogenetic basis of phototaxis.
\end{abstract}

KEY WORDS: phototaxis; Drosophila; correlated response; selection; sepia.

\section{INTRODUCTION}

Phototaxis is a complex behavioral response involving several components of perception and neurological processing. Light must first be absorbed by the receptor cells and elicit neural excitation. These signals must be transmitted across synaptic junctions to points where the information can be integrated and processed in the central nervous system. When at last motor signals are generated, the end point of the behavioral response can be scored as a motor response (Benzer, 1967).

In genetically heterogeneous populations of Drosophila, selection for phototactic behavior can be very effective (Hadler, 1964; Markow, 1975a; Köhler, 1977). The genetic basis for this response to selection can involve loci whose phenotypic effects lie in any of the components outlined above. An understanding of the neurogenetic basis of the behavior requires an identification of the affected component and a clarification of the mech-

This work was supported by NIH Grants GM19583, GM25424, and NS15263.

${ }^{1}$ Department of Zoology, Arizona State University, Tempe, Arizona 85287.

2 Department of Biology, Pennsylvania State University, University Park, Pennsylvania 16802 . 
anism by which the genetic lesion results in aberrant visual processing. Behavioral tests that selectively screen for defects in different components of visual response have been effective in identifying a number of specific mutants (Heisenberg and Götz, 1975).

Another approach in exploring the neurogenetics of behavior is to examine the behavior of mutants for which there is reason to believe a component of visual perception is affected. Eye color mutants of $D$. melanogaster have received the most attention, because altered screening pigments can be easily related to visual perception. Approaches have included scoring phototaxis (Fingerman, 1952; Markow and Scavarda, 1977; Köhler, 1977; Köhler et al., 1980), examining optomotor response (Kalmus, 1943; Wehner et al., 1969), and measuring electroretinograms (Pak et al., 1969; Hotta and Benzer, 1969). In this study selection for phototactic behavior was performed on populations of $D$. melanogaster that were segregating at the sepia locus. The observed correlated response strengthens the notion that phototactic selection response is at least partially mediated by the effects on visual perception of screening pigmentation.

\section{MATERIALS AND METHODS}

Experimental Populations. Three experimental populations of $D$. melanogaster were constructed, each polymorphic for the third-chromosome recessive mutant sepia (3-26.0). The wild-type strain used was originally created in 1976 by combining 200 flies from 12 wild-type strains in a population cage (Markow, 1975a). The sepia strain was obtained from the Drosophila Stock Center at the California Institute of Technology. The $F_{2}$ generation from the cross between the wild and the sepia strains was used to initialize the selected populations with a sepia allelic frequency of 0.5 .

Selection for Phototactic Behavior. The Hirsch-Hadler mazes employed in selecting for phototactic behavior have been described extensively elsewhere (Markow, 1975a). Flies enter a maze through a starting tube and make a series of 15 light/dark choices provided by $\mathrm{Y}$ tubes. After completing the maze, flies emerge in 16 collecting tubes. Those individuals emerging in tube No. 1 have made all dark choices, while flies making all light choices appear in tube No. 16. The numbers of flies in the 16 collecting tubes define the photoscore distribution, whose mean may range from 1 (highly photonegative) to 16 (highly photopositive). A photoneutral population has a mean photoscore of 8.5. Mazes were thoroughly washed and dried after each trial, and randomization of mazes was done to prevent a "maze effect." 
From the $F_{2}$ generation, virgin males and females were separated under light ether anesthesia and stored until 4 days old. Five hundred females and five hundred males were tested in separate mazes. The most photopositive 100 pairs were placed in a population cage to found a photopositive selection line. The most photonegative 100 pairs were used to start a photonegative line. A control line was started with 100 pairs of unselected flies. Three replicates of each of these three treatments were established. Every generation, 500 flies of each sex were tested in mazes, and 100 of the most extreme flies were selected as parents of the next generation. The control lines were always tested in mazes, but new generations were begun with 100 untested, random pairs of flies. Selected and control populations were reared with discrete generations in population cages containing 12 cups of cornmeal-molasses-agar medium.

Estimation of sepia Allelic Frequency. The frequency of the se allele was determined in all treatments by performing progeny tests at generations 0,7 , and 12 . Three hundred virgin females from each experimental and each control line were selected at random and scored for eye color phenotype. The number of females with sepia eyes was recorded and these females were discarded. The remaining females were mated individually to homozygous sepia males, and genotypes were inferred from the progeny. This procedure allowed accurate estimation of genotypic frequencies.

\section{RESULTS}

Selection for Phototaxis. Photoscores appeared to diverge markedly between photopositively and photonegatively selected treatments (Fig. 1), but in order to ascertain the statistical significance of this trend, the distributions of photoscores must be characterized. Figure 2 presents a histogram of the founding population and shows how this distribution becomes skewed as the means shift. If every light/dark decision made by the flies were independent of previous choices (and other flies), photoscores would be distributed binomially. In every case the fit to a binomial distribution was very poor, because scores were overrepresented in the tails of the distribution (see also Stark et al., 1981). Failure to fit the binomial distribution suggests either a lack of independence of successive choices by individual flies or a tendency of flies to avoid more heavily traveled routes.

Before performing an analysis of variance, the assumption of normality was tested with the Kolmogorov-Smirnov test. Only 13 of the 36 untransformed photoscore distributions gave adequate fits to the normal distribution, while log transformation removed some skewness and 25 of 


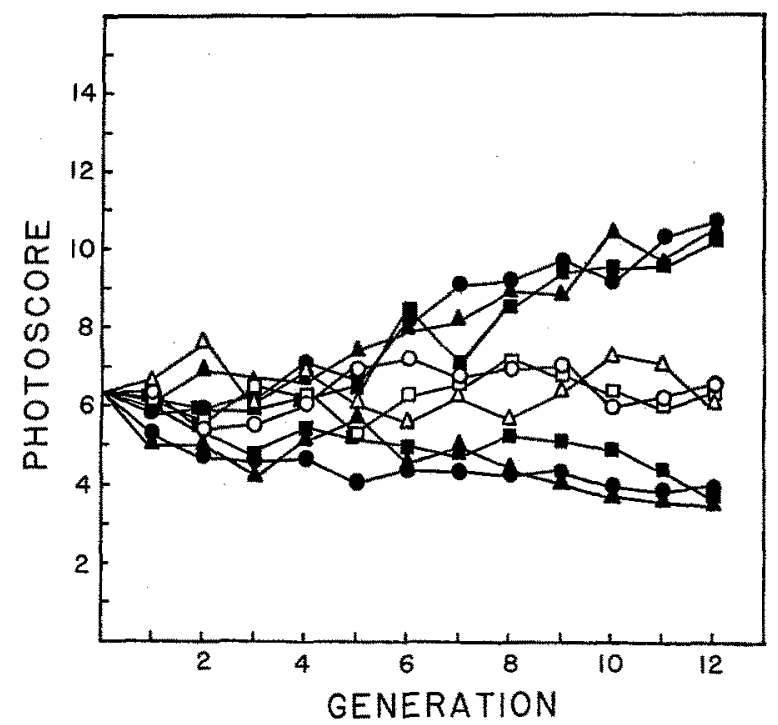

Fig. 1. Response to photoscore selection. Mean photoscores of pooled sexes are plotted for each of three replicates of photopositively selected lines (upper three curves), controls (open figures), and photonegatively selected lines (lower three curves).

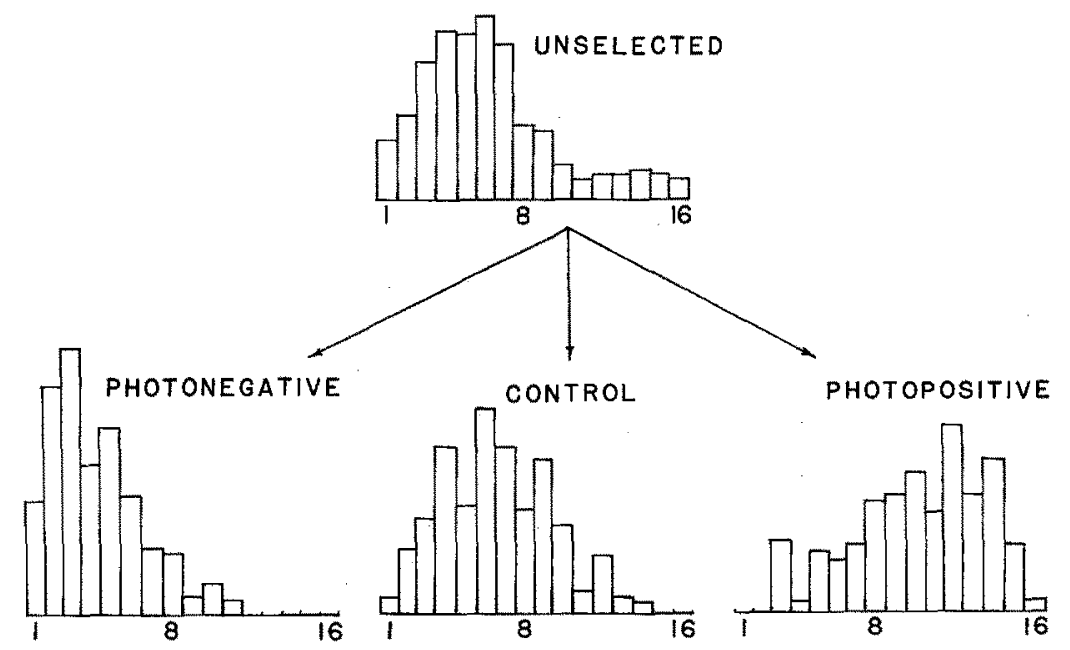

Fig. 2. Typical frequency distributions of photoscores in the founding population and after 12 generations of each treatment. 
Table I. Photoscores and sepia Allele Frequencies ${ }^{a}$

\begin{tabular}{|c|c|c|c|c|c|c|c|c|}
\hline \multirow[b]{3}{*}{ Population } & \multicolumn{4}{|c|}{ Generation 7} & \multicolumn{4}{|c|}{ Generation 12} \\
\hline & \multicolumn{3}{|c|}{ Mean photoscore } & \multirow[b]{2}{*}{$a$} & \multicolumn{3}{|c|}{ Mean photoscore } & \multirow[b]{2}{*}{$q$} \\
\hline & Female & $t$ & Male & & Female & $t$ & Male & \\
\hline \multicolumn{9}{|l|}{ Control } \\
\hline 1 & $6.64 \pm 0.15$ & & $8.87 \pm 0.18$ & 0.17 & $5.62 \pm 0.18$ & $* * *$ & $6.54 \pm 0.19$ & 0.15 \\
\hline 2 & $6.08 \pm 0.19$ & $* * *$ & $4.97 \pm 0.24$ & 0.05 & $5.13 \pm 0.21$ & $* * *$ & $3.72 \pm 0.13$ & 0.02 \\
\hline 3 & $6.54 \pm 0.21$ & $* *$ & $6.02 \pm 0.20$ & 0.26 & $6.24 \pm 0.19$ & & $6.00 \pm 0.16$ & 0.12 \\
\hline \multicolumn{9}{|c|}{ Photopositive } \\
\hline 1 & $9.32 \pm 0.22$ & & $9.15 \pm 0.22$ & 0.33 & $11.79 \pm 0.23$ & & $11.60 \pm 0.20$ & $0.24 *$ \\
\hline 2 & $8.20 \pm 0.19$ & & $8.31 \pm 0.27$ & 0.34 & $9.52 \pm 0.22$ & ** & $10.18 \pm 0.22$ & 0.32 \\
\hline 3 & $8.98 \pm 0.13$ & $* * *$ & $7.93 \pm 0.16$ & 0.59 & $10.50 \pm 0.18$ & & $10.18 \pm 0.20$ & 0.63 \\
\hline \multicolumn{9}{|c|}{ Photonegative } \\
\hline 1 & $4.02 \pm 0.17$ & $* * * *$ & $4.80 \pm 0.20$ & 0.19 & $3.57 \pm 0.14$ & & $3.41 \pm 0.14$ & $0.14^{*}$ \\
\hline 2 & $5.42 \pm 0.16$ & **** & $4.69 \pm 0.16$ & 0.27 & $4.09 \pm 0.15$ & $* * *$ & $3.00 \pm 0.15$ & $0.10^{*}$ \\
\hline 3 & $4.99 \pm 0.17$ & $* * *$ & $4.11 \pm 0.17$ & $0.20^{*}$ & $4.72 \pm 0.17$ & 冰头 & $3.11 \pm 0.17$ & $0.10^{*}$ \\
\hline
\end{tabular}

${ }^{a}$ Means and standard errors of photoscore are given for each sex, with the significance of the $t$ test for each pair indicated between them. Estimates of allele frequency are given, with significance of goodness of fit to Hardy-Weinberg indicated by asterisks.

$* P<0.05$.

** $P<0.01$.

*** $P<0.001$.

the 36 distributions fit normality. Homogeneity of variances was tested, and the ratio of the largest to the smallest variance among the 36 photoscores was well below the critical $5 \%$ value $\left(F_{\max }=4.02\right)$.

Heterogeneity between sexes was investigated by performing two sample $t$ tests on mean male and female photoscores. The results, given in Table $I$, indicate that 11 of 18 tests were significant. In 9 of these 11 cases the females had a higher mean photoscore than the males. These differences preclude pooling the female and male photoscores in subsequent statistical tests.

Nested analysis of variance was used to determine whether replicates within a treatment were homogeneous and whether there was a treatment effect. Table II presents the results of one-way fixed effects ANOVA on $\log$-transformed photoscores for generations 7 and 12 in males and females. In all cases there is a significant heterogeneity among replicates within treatments, and the phototactic selection treatments also caused significant differences. Two-way analysis of variance yielded significant sex and treatment effects, as well as significant sex $\times$ treatment interactions. This is consistent with the $t$ tests mentioned above. Scheffé's à posteriori multiple comparison test indicates that in both sexes, all three 
Table II. Photoscore Analysis of Variance ${ }^{a}$

\begin{tabular}{lrrrr}
\hline & df & SS & MS & \multicolumn{1}{c}{$F$} \\
\hline Generation 7 & & & & \\
$\quad$ Females & 2 & 292.56 & 146.28 & $22.30^{* * *}$ \\
$\quad$ Treatments & 6 & 39.35 & 6.56 & $16.39^{* * *}$ \\
$\quad$ Replicates within treatments & 4454 & 1782.4 & 0.40 & \\
$\quad$ Within & 4462 & 2114.32 & & \\
$\quad$ Total & 2 & 419.61 & 209.80 & $20.49^{* *}$ \\
Males & 6 & 61.46 & 10.24 & $20.48^{* * *}$ \\
$\quad$ Treatments & 4475 & 2242.20 & 0.50 & \\
$\quad$ Replicates within treatments & 4483 & 2723.27 & & \\
$\quad$ Within & & & & \\
$\quad$ Total & 2 & 797.34 & 398.67 & $59.62^{* * *}$ \\
Generation 12 & 6 & 40.12 & 6.69 & $17.61^{* * *}$ \\
Females & 4425 & 1680.55 & 0.38 & \\
$\quad$ Treatments & 4433 & 2518.01 & & \\
$\quad$ Replicates within treatments & & & \\
$\quad$ Within & 2 & 1188.06 & 594.03 & $46.37^{* * *}$ \\
$\quad$ Total & 6 & 76.87 & 12.81 & $28.09^{* * *}$ \\
Males & 4435 & 2021.51 & 0.46 & \\
$\quad$ Treatments & 3286.44 & & \\
$\quad$ Replicates within treatments & 4443 & & \\
$\quad$ Within & & & \\
$\quad$ Total &
\end{tabular}

${ }^{a}$ Nested one-way analysis of variance of log-transformed photoscore data. Sexes and generations are treated separately because they were found to be heterogeneous.

treatments were significantly different from one another. In conclusion, there was a clear response to photoscore selection, and by generation 12 strongly divergent phototactic behavior was observed.

Realized heritability was estimated in lines undergoing photopositive and photonegative selection according to the method of Falconer (1960). The generation mean photoscores were plotted against the cumulated selection differentials, and the realized heritability was estimated by the regression coefficient. Standard errors were estimated following the method of Hill (1972). The heritabilities realized over 12 generations of photonegative selection were $0.11,0.10$, and 0.08 for the three replicates, giving a pooled value of $0.10 \pm 0.009$. Among the photopositive lines the realized heritabilities were $0.07,0.07$, and 0.09 , giving a pooled value of $0.08 \pm 0.007$.

Frequency of sepia. Figure 3 presents the changes in sepia allelic frequency at generation 7 and 12 in all nine populations. The significance of changes in sepia frequency was examined by (1) Monte Carlo computer simulations and (2) analysis of variance-covariance matrices after the fashion of Schaffer et al. (1977) and Wilson (1980). A pure drift model 


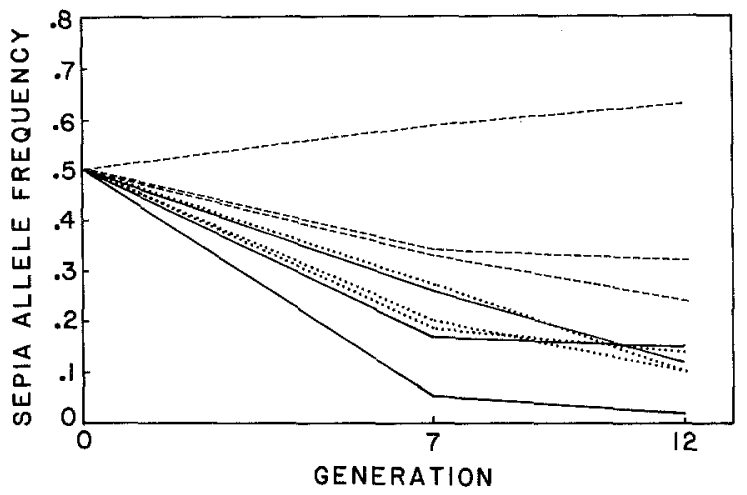

Fig. 3. sepia allele frequency at generations 0,7 , and 12 in three replicates of each of three treatments. Solid lines represent controls, dashed lines represent photopositively selected populations, and dotted lines represent photonegatively selected populations.

cannot be used to ascertain among-replicate differences, because the sepia allelic frequency follows a clear declining trend. Rather, a selection model must be superimposed onto the sampling procedure. Furthermore, since the strength of drift depends strongly on sample size, the effective population size must be considered. Previous investigators (Kerr and Wright, 1954a,b; Wright and Kerr, 1954; Buri, 1956; Crow and Morton, 1955) have determined that a reasonable approximation to the effective population size of Drosophila is half the observed population size.

The Monte Carlo procedure for estimating the empirical allelic frequency distribution was performed by trinomial sampling of adult genotypes after selection. The selection model used was the classical onelocus viability model:

$$
q^{\prime}=\frac{w_{12} p q+w_{22} q^{2}}{w_{11} p^{2}+w_{12} 2 p q+w_{22} q^{2}},
$$

where $q$ is the frequency of the sepia allele, and the fitnesses of $+1+$, $s e /+$, and se/se genotypes are $w_{11}, w_{12}$, and $w_{22}$, respectively. Three oneparameter fitness models were used:

(1) multiplicative $-w_{11}=1, w_{12}=s, w_{22}=s^{2}$;

(2) additive $-w_{11}=1, w_{12}=1-s, w_{22}=1-2 s$; and

(3) complete dominance- $w_{11}=w_{12}=1, w_{22}=s$.

There are not sufficient degrees of freedom to obtain direct maximum-likelihood fitness estimates from the data. Approximate fitness estimates were made by empirically finding trajectories of allelic frequency passing through the generation 7 or 12 mean allelic frequency. The $95 \%$ 
Table III. Monte Carlo Simulation Results ${ }^{a}$

\begin{tabular}{|c|c|c|c|c|c|c|}
\hline & \multicolumn{3}{|c|}{ Generation 7} & \multicolumn{3}{|c|}{ Generation 12} \\
\hline & \multirow{2}{*}{ Fitnesses } & \multicolumn{2}{|c|}{ Confidence intervals } & \multirow{2}{*}{ Fitnesses } & \multicolumn{2}{|c|}{ Confidence intervals } \\
\hline & & $N^{\mathrm{e}}=100$ & $N_{\mathrm{e}}=200$ & & $N_{e}=100$ & $N_{\mathrm{e}}=200$ \\
\hline \multirow[t]{4}{*}{ Control } & $M(1,0.80,0,64)$ & $0.072-0.320$ & $0.111-0.257$ & $\mathrm{M}(1,0.83,0.69)$ & $0.036-0.207$ & $0.041-0.182$ \\
\hline & $\mathrm{A}(1,0.81,0.62)$ & $0.068-0.319$ & $0.109-0.251$ & $A(1,0.84,0.68)$ & $0.039-0.215$ & $0.043-0.186$ \\
\hline & $\mathrm{D}(1,1,0.30)$ & $0.085-0.246$ & $0.103-0.210$ & $D(1,1,0.26)$ & $0.044-0.181$ & $0.051-0.152$ \\
\hline & Observed sepia & $\begin{array}{l}\text { frequencies: } \\
0.26\end{array}$ & $0.17,0.05$ & Observed sepia & $\begin{array}{l}\text { frequencies: } \\
0.12\end{array}$ & $0.15,0.02$ \\
\hline \multirow[t]{4}{*}{ Photopositive } & $\mathrm{M}(1,0.96,0.92)$ & $0.250-0.611$ & $0.311-0.565$ & $\mathrm{M}(1,0.97,0.94)$ & $0.186-0.623$ & $0.255-0.605$ \\
\hline & A $(1,0.96,0.92)$ & $0.248-0.605$ & $0.309-0.563$ & A $(1,0.97,0.94)$ & $0.194-0.630$ & $0.243-0.590$ \\
\hline & D $(1,1,0.91)$ & $0.275-0.577$ & $0.322-0.548$ & $D(1,1,0.92)$ & $0.185-0.610$ & $0.248-0.515$ \\
\hline & Observed sepia & $\begin{array}{l}\text { frequencies: } \\
0.59\end{array}$ & $0.33,0.34$ & Observed sepia & $\begin{array}{l}\text { frequencies: } \\
0.63\end{array}$ & $0.24,0.32$ \\
\hline \multirow[t]{4}{*}{ Photonegative } & $\mathrm{M}(1,0.84,0.70)$ & $0.106-0.372$ & $0.146-0.334$ & $\mathrm{M}(1,0.85,0.72)$ & $0.039-0.261$ & $0.057-0.232$ \\
\hline & A $(1,0.85,0.70)$ & $0.111-0.374$ & $0.153-0.339$ & A $(1,0.85,0.70)$ & $0.035-0.263$ & $0.051-0.230$ \\
\hline & $\mathrm{D}(1,1,0.53)$ & $0.145-0.330$ & $0.148-0.278$ & $\mathrm{D}(1,1,0.39)$ & $0.047-0.209$ & $0.060-0.192$ \\
\hline & Observed sepia & $\begin{array}{l}\text { frequencies: } \\
0.20\end{array}$ & $0.19,0.27$ & Observed sepia & $\begin{array}{l}\text { frequencies: } \\
0.10\end{array}$ & $0.14,0.10$ \\
\hline
\end{tabular}

${ }^{a}$ Observed sepia allele frequencies for the three replicates are given, along with fitnesses of $(+/+, s e /$ ,$+ s e / s e$ ) which give a trajectory passing through the mean frequency. The three fitness models were as follows: $\mathrm{M}$, multiplicative $\left(w_{11}=1, w_{12}=s, w_{22}=s^{2}\right) ; \mathrm{A}$, additive $\left(w_{11}=1, w_{12}=1-s, w_{22}\right.$ $=1-2 s)$; and $\mathrm{D}$, complete dominance $\left(w_{11}=w_{12}=1, w_{22}=s\right)$. For each fitness set, 1000 populations were sampled numerically to yield the given $95 \%$ empirical confidence intervals.

confidence intervals for the allelic frequencies at generations 7 and 12 were constructed based on 1000 populations for each of three fitness sets for each treatment. Results are tabulated in Table III. Even with an effective population size of 100 , the control replicates failed to fall within their expected confidence intervals. This is partly due to the unusually low frequency of sepia in one replicate and the fact that the confidence intervals were constructed about the mean frequency. The observed range of control sepia frequencies does not exceed the expected confidence interval. Nevertheless, the data suggest that within-treatment heterogeneity is somewhat greater than would be expected due to drift alone. The within-treatment heterogeneity is small compared to the differences among treatments in expected confidence intervals and observed sepia ranges. We conclude that the different treatments of phototactic selection resulted in significantly different sepia allelic frequencies.

A variance-covariance matrix procedure, recently presented by Schaffer et al. (1977) and Wilson (1980), was used to obtain chisquare values for the goodness of fit of observed to expected allelic frequencies. Three hypotheses were tested with this procedure: (1) $H_{0}$-drift occurs 
Table IV. Drift Analysis Using Variance-Covariance Matrices ${ }^{a}$

\begin{tabular}{lccc}
\hline & df & $N_{\mathrm{e}}=\frac{1}{2} N$ & $N_{\mathrm{e}}=N$ \\
\hline$H_{0} P=0.5$ & & & \\
$\quad$ Control & 6 & $72.58^{*}$ & $137.04^{* *}$ \\
$\quad$ Photopositive & 6 & 10.50 & $19.61^{*}$ \\
$\quad$ Photonegative & 6 & $47.95^{*}$ & $88.52^{* *}$ \\
$\quad$ Sum & 18 & $131.03^{*}$ & $245.17^{* * *}$ \\
$H_{1} \quad$ & & \\
$\quad$ Linear trend & & $19.02^{*}$ & $33.69^{* *}$ \\
Control & 5 & $20.14^{*}$ & $19.61^{*}$ \\
Photopostive & 5 & $12.38^{*}$ & $19.29^{*}$ \\
Photonegative & 5 & $51.54^{*}$ & $77.59^{*}$ \\
$\quad$ Sum & 15 & & \\
$H_{2} \quad$ & 12.12 & $20.54^{*}$ \\
$\quad$ Different linear trends & & 7.80 & $14.40^{*}$ \\
Control & 5 & 11.62 & $17.71^{*}$ \\
Photopositive & 5 & $31.54^{*}$ & $52.65^{*}$ \\
Photonegative & 5 & & \\
$\quad$ Sum & 15 & & \\
\hline
\end{tabular}

${ }^{a}$ Chi-square values for goodness of fit to three sampling models. Asterisks indicate significance of heterogeneity among replicates.

about $P=0.5 ;(2) H_{i}$-drift occurs about a linear trend in allelic frequency that is common to all treatments; and (3) $H_{2}$-drift occurs about different linear trends in the three treatments. Results are presented in Table IV. Again it is clear that the different treatments are associated with significantly different sepia allelic frequencies.

Using estimates of genotypic frequencies, goodness of fit to HardyWeinberg proportions was tested. As shown in Table I, 5 of 18 tests failed to fit the expected Hardy-Weinberg proportions, and 4 of these 5 occurred at generation 12. Moreover, all of the controls gave adequate fits, while one of six tests of photopositive selected lines failed to fit, and four of six tests of photonegative selected lines failed to fit.

Correlated Effects. An important control to consider is the photoscores of the unselected flies of the different sepia genotypes. Markow and Scavarda (1977) derived strains of Drosophila with the se and $s e^{+}$ allele replaced in a coisogenic Canton-S background. These data are useful in determining the extent to which se is the cause of altered photobehavior. Two replicates of each genotype were tested, and results reported are means $\pm \mathrm{Se}$, with sample sizes given in parentheses. The photoscores were $7.43 \pm 0.22(261)$ and $7.37 \pm 0.15$ (534) for se/se females, $7.16 \pm 0.35(139)$ and $6.45 \pm 0.29(165)$ for $s e /+$ females, and 

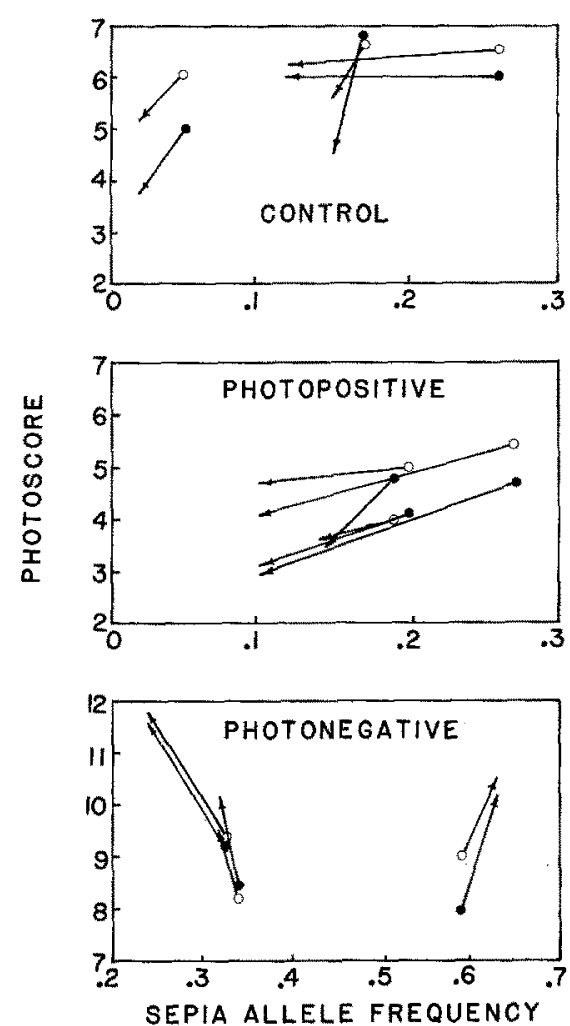

Fig. 4. Coordinate changes in mean photoscore and sepia allele frequency. The tail of each arrow represents the mean photoscore and allele frequency at generation 7 , while the head represents values at generation 12 . Three replicates are depicted on each graph, with open circles representíng females and filled circles representing males.

$6.39 \pm 0.30(160)$ and $4.89 \pm 0.28(141)$ for $+/+$ females. Even among unselected flies, the se genotypes appear to be more photopositive.

Figure 4 is a graphical presentation of the relationship between sepia allelic frequency and mean photoscore in the transitions from generation 7 to generation 12 . These graphs clearly depict the strong correlation between sexes in mean photoscore, and Spearman rank correlation coefficients were 0.88 at generation 7 and 0.95 at generation 12 . More importantly, since the arrows are roughly parallel within each plot, this indicates that the relationship between photoscore and sepia frequency is consistent. In addition, the different treatments cause clearly different responses in these phase plots. These data cannot simply be pooled onto a pair of axes to calculate a correlation, since different observations of 
the same population are not independent. Correlations can, however, be calculated across treatments within each sex and generation. For females at generations 7 and 12 the photoscore-sepia frequency Spearman rank correlation coefficients were 0.60 (ns) and $0.68(P<0.05)$, while for males these correlations were 0.55 (ns) and $0.77(P<0.01)$.

\section{DISCUSSION}

Selection for Phototaxis. Selection for photopositive and photonegative behavior produced gradual divergence in photoscores characteristic of traits controlled by genes acting in an additive manner. Despite the caveat that measures of phototaxis are highly context dependent (Rockwell and Sieger, 1973; Grossfield, 1978), the realized heritabilities were quite similar to those previously reported in D. melanogaster (Markow, 1975a; Köhler, 1977) as well as in other species (Dobzhansky and Spassky, 1967; Markow and Smith, 1977, 1979).

The observed tendency of males to be more photonegative than females has consistently been observed in $D$. melanogaster. These sex differences in photoscore may be due to sex-specific expression of autosomal genes or sex linkage of genes controlling phototactic behavior. Major sex-linked genes affecting phototaxis have been identified in $D$. melanogaster (Markow, 1975a,b; Köhler, 1977).

Frequency of sepia. Two different methods have been used to show that it is exceedingly unlikely that the differences in sepia frequency seen among the nine populations could be due to drift alone. The robustness of the results of the Monte Carlo simulations was tested using a variety of selection schemes and two different effective population sizes. Although it is known that the mating success of sepia and wild-type males are not the same (Markow, unpublished data), it is unlikely that simulations allowing these differences would have yielded significantly different empirical confidence intervals. Viabilities were arbitrarily chosen to give gene frequency trajectories going through the observed mean frequencies, and models allowing differences in mating success can be fitted to the data with equal precision. The complete dominance model had fitnesses different by a factor of two from the additive and multiplicative models, yet the empirical confidence intervals (the strength of drift) were similar. The intervals were somewhat smaller under the model of complete dominance, and this is undoubtedly due to the fact that the binomial variance depends on the frequency. Since sepia homozygotes were rarer after selection under the complete dominance model, the sampling variance was lower. 
Heterogeneity among replicates within a treatment was detected by observing that not all replicates fell within the respective empirical confidence interval. Despite this, it remains clear that the heterogeneity among treatments is much greater than the heterogeneity among replicates within a treatment. Additional error is undoubtedly caused by such experimental problems as heterogeneity in environmental conditions.

The analysis of variance-covariance matricies gave similar results: not all replicates fit the model of drift about $P=0.5$, nor do they fit the same linear trend. When different linear trends are allowed for each treatment, most of the within treatment heterogeneity disappeared. Among the control populations, there was a highly significant improvement in fit by allowing a linear trend $\left(\chi^{2}=72.58-12.12=60.46,1 \mathrm{df}\right)$, suggesting strong selection against sepia in the absence of phototactic selection.

The asymmetry of response in sepia frequency was striking. The photopositive lines maintained a sepia frequency of about 0.5 , whereas both the controls and the photonegative lines declined in sepia frequency approximately to the same extent. One explanation is that natural selection in the same direction (photonegative) does not significantly accelerate this process due to a loss of genetic variance. Photopositive selection, on the other hand, may be opposing the natural selection against sepia. Second, partial dominance at the sepia locus could lead to an asymmetry in response. Third, as photonegative selection proceeds, more flies cluster in the most photonegative tubes, so the selection differential decreases. Since the selection differential decreases as the experiment proceeds in the photonegative direction, but not as much in the photopositive direction, the photonegative selection is expected to deviate less from the control.

Testing goodness of fit to Hardy-Weinberg proportions is a rather crude means of detecting natural selection, since deviations are neither necessary nor sufficient to prove the existence of selective differences (Lewontin and Cockerham, 1959). The test gains meaning, however, when comparisons among treatments are made. The control populations never significantly deviated from Hardy-Weinberg genotypic proportions, while among the photopositive populations there was one significant deviation at generation 12. Among photonegative populations, one replicate failed to fit at generation 7 , and all three replicates showed significant deviation from Hardy-Weinberg proportions in generation 12. Hence it is clear that there are differences in genotypic distributions among the three treatments. It is especially interesting that the controls and photonegative populations are different in genotypic distributions yet have approximately the same sepia allelic frequencies. 
Correlated Response. It is clear that the photopositively selected populations retained a higher frequency of sepia than photonegative populations and controls. The photoscores of unselected se/se and se/ + flies (Markow and Scavarda, 1977) suggest a causal connection between phototactic selection and the correlated sepia frequency response. Without this kind of prior knowledge, it would not be appropriate to conclude that the correlated response was causally related. Pasteur (1969) observed a number of morphological changes among flies selected for phototaxis and geotaxis, but whether the morphological differences were inherent to the altered behavior is unclear. Stuber et al. (1980) observed correlated response at allozyme loci among lines of maize selected for grain yield. Here again, there is no reason to conclude that the differences in yield were mediated by the allozyme differences. Instead, other loci linked to the allozyme loci may be responsible for the yield differences.

One line of evidence supporting a causal relation between correlated responses is a mechanistic relationship between the selected locus and the quantitative character. In the present context, there is a wealth of evidence suggesting that mutants affecting visual screening pigments also affect photoresponse in Drosophila. One class of tests that demonstrates the role of screening pigments measures the optomotor response (Kalmus, 1943; Wehner et al., 1969). These studies have shown that mutants lacking screening pigments in their eyes are less able to orient precisely relative to dark and light fields. The conclusion is that screening pigments are necessary for contrast perception and visual acuity. Burnet et al. (1968) further demonstrated the role of screening pigments by feeding $v ; b w$ mutant flies kynurenine. The $v ; b w$ flies normally have a white eye phenotype and poor optomotor response, but kynurenine in the diet restored pigmentation to the eye as well as improving visual acuity.

Results of tests of phototactic behavior suggest that optomotor response is relevant to phototactic selection experiments. Fingerman (1952), Markow and Scavarda (1977), Köhler (1977), and Köhler et al. (1980) observed that flies with less visual pigment tend to be less photopositive. This may be due either to their poor visual acuity or to an avoidance of high light intensity. Fingerman (1952) observed that white mutants become more photopositive when tested in dim light, and the issue of adaptation to light intensity may be a serious problem in short-term (less that a few minutes) phototactic tests (Grossfield, 1978).

The experiments of Köhler (1977) and Köhler et al. (1980) represent the converse of the experiments reported here. They observed fixation of the mutants white, garnet, and vermilion in polymorphic populations subjected to photonegative selection, while the frequencies of alleles for scute, cut, and forked (none of which are eye color mutants) were not 
affected by phototactic selection. When this is considered in light of our results, it appears that the altered photoresponse of eye pigment mutants makes these loci targets for phototactic selection.

The correlation between eye pigmentation and photobehavior may be relevant in a broader evolutionary context. Among Drosophila species, there is extensive variation in both eye pigmentation and photoresponse (Grossfield 1978). If photobehavior is important in some aspect of ecological adaptation, and if the behavior is genetically heritable, then such adaptation can be described as selection for behavioral changes driving the correlated morphological change in eye pigmentation. We will never know the extent to which variation in eye pigmentation among Drosophila species is due to selection for photobehavior, but the correlated responses seen in this study and those of Köhler (1977) and Köhler et al. (1980) are suggestive.

It would be premature to suggest that all response to phototactic selection is mediated by genes affecting visual physiology. In addition to perception of light, photobehavior entails a complex set of neural processes coupled to a locomotor response. Electroretinograms of nonphototactic mutants are generally normal (Pak et al., 1969), suggesting that at least the ommatidial cells are functioning. But it is still possible that there are mutants with lesions in neurons that carry visual signals to the brain. It is clear that a full understanding of photobehavior will require genetic analysis of neurophysiology at the individual level.

\section{ACKNOWLEDGMENTS}

The authors thank Brenda Holloway, Johnna Sovola, and Billie Grounds for technical assistance.

\section{REFERENCES}

Benzer, S. (1969). Behavioral mutants of Drosophila isolated by countercurrent distribution. Proc. Natl. Acad. Sci. 58:1112-1119.

Buri, P. (1956). Gene frequency in small populations of mutant Drosophila. Evolution 10:367402.

Burnet, B., Connolly, K., and Beck, J. (1968). Phenogenetic studies on visual acuity in $D$. melanogaster. J. Insect Physiol. 14:855-866.

Crow, J. F., and Morton, N. E. (1955). Measurements of gene frequency drift in small populations. Evolution 9:202-214.

Dobzhansky, T. H., and Spassky, B. (1967). Effects of selection and migration on geotactic and phototactic behavior of Drosophila I. Proc. R. Soc. Lond. 168:27-47.

Falconer, D. S. (1960). Introduction to Quantitative Genetics, Ronald Press, New York.

Fingerman, M. (1952). The role of eye pigments of Drosophila melanogaster in photic orientation. J. Exp. Zool. 120:131-164.

Grossfield, J. (1978). Nonsexual behavior of Drosophila. In Ashburner, M., and Wright, T. R. F. (eds.), The Genetics and Biology of Drosophila, Vol. $2 b$, pp. 1-26. 
Hadler, N. (1964). Heritability and phototaxis in Drosophila melanogaster. Genetics 50:1269-1277.

Heisenberg, M., and Götz, K. G. (1975). The use of mutations for the partial degradation of vision in Drosophila melanogaster. J. Comp. Physiol. 98:217-241.

Hill, W. G. (1972). Estimation of realized heritabilities from selection experiments. I. Divergent selection. Biometrics 28:747-765.

Hotta, Y., and Benzer, S. (1969). Abnormal electroretinograms in visual mutants of Drosophila. Nature 222:354-356.

Kalmus, H. (1943). The optomotor response of some eye mutants of Drosophila. J. Genet. 45:206-214.

Kerr, W. E., and Wright, S. (1954a). Experimental studies of the distribution of gene frequencies in very small populations of $D$. melanogaster. I. Forked. Evolution 8:172177 .

Kerr, W. E., and Wright, S. (1954b). Experimental studies of the distribution of gene frequencies in very small populations of $D$. melanogaster. III. Aristapedia and spineless. Evolution 8:293-302.

Köhler, W. (1977). Investigations on the phototactic behavior of Drosophila melanogaster. I. Selection response in the presence of a multiply marked X-chromosome. Genetica 47:93-100.

Köhler, W., Krause, J., and Michutta, A. (1980). Heritability of phototactic behavior of Drosophila. Dros. Inform. Serv. 55:77-79.

Lewontin, R. C., and Cockerham, C. C. (1959). The goodness of fit test for detecting natural selection in random mating populations. Evolution 13:561-564.

Markow, T. A. (1975a). A genetic analysis of phototactic behavior in D. melanogaster. I. Selection in the presence of inversions. Genetics 79:527-534.

Markow, T. A. (1975b). A genetic analysis of phototactic behavior in D. melanogaster. II. Hybridization of divergent strains. Behav. Genet. 5:339-350.

Markow, T. A., and Scavarda, N. J. (1977). Effect of age and of screening pigment mutations on the phototactic behavior of Drosophila melanogaster. Behav. Genet. 7:139-146.

Markow, T. A., and Smith, W. L. (1977). Genetic analysis of phototactic behavior of Drosophila simulans. Genetics 85:273-278.

Markow, T. A., and Smith, W. L. (1979). Genetics of phototactic behavior in D. ananassae, a member of the melanogaster group. Behav. Genet. 9:61-67.

Pasteur, G. (1969). Morphological differences between Drosophila pseudoobscura selected for opposite geotaxes and phototaxes. Genetics 62:837-847.

Rockwell, R. F., and Sieger, M. B. (1973). Phototaxis in Drosophila: A critical review. Am. Sci. 61:339-345.

Schaffer, H. E., Yardley, D., and Anderson, W. W. (1977). Drift or selection: A statistical test of gene frequency variation over generations. Genetics 87:371-379.

Stark, W. S., Hu, K. G., and Srygley, R. B. (1981). Comparisons of phototaxis properties in different mazes. Dros. Inform. Serv. 56:131-132.

Stuber, C. W., Moll, R. H., Goodman, M. M., Schaffer, H. E., and Weir, B. S. (1980). Allozyme frequency changes associated with selection for increased grain yield in maize (Zea mays). Genetics 95:225-236.

Wehner, R., Gartenmann, G., and Jungi, T. (1969). Contrast perception in eye color mutants of D. melanogaster and D. subobscura. J. Insect Physiol. 15:815-823.

Wilson, S. R. (1980). Analyzing gene frequency data when the effective population size is finite. Genetics 95:489-502.

Wright, S., and Kerr, W. E. (1954). Experimental studies of the distribution of gene frequencies in very small populations of Drosophila melanogaster II. Bar. Evolution 95:225-236. 